\author{
EUROPEAN ORGANIZATION FOR NUCLEAR RESEARCH \\ European Laboratory for Particle Physics
}

\title{
DESIGN, PRODUCTION AND FIRST COMMISSIONING RESULTS OF THE ELECTRICAL FEEDBOXES OF THE LHC
}

\author{
A. Perin ${ }^{1}$, S. Atieh ${ }^{1}$, V. Benda ${ }^{1}$, A. Bertarelli ${ }^{1}$, A. Bouillot ${ }^{1}$, K. Brodzinski ${ }^{3}$, R. Folch ${ }^{1}$, J. Fydrych ${ }^{3}$, \\ M. Genet' ${ }^{1}$, S. Koczorowski ${ }^{1}$, L. Metral' ${ }^{1}$, J. Miles ${ }^{1}$, F. Millet', K. Polkovnikov'², R. Rabehl' ${ }^{4}$, \\ L. Serio ${ }^{1}$, V. Sytchev ${ }^{2}$, V. Sytnik ${ }^{2}$, P. Trilhe ${ }^{1}$, R. van Weelderen ${ }^{1}$, V. Zhabitskiy ${ }^{2}$
}

\begin{abstract}
A total of 44 CERN designed cryogenic electrical feedboxes are needed to power the LHC superconducting magnets. The feedboxes include more than 1000 superconducting circuits fed by high temperature superconductor and conventional current leads ranging from $120 \mathrm{~A}$ to $13 \mathrm{kA}$. In addition to providing the electrical current to the superconducting circuits, they also ensure specific mechanical and cryogenic functions for the LHC. The paper focuses on the main design aspects and related production operations and gives an overview of specific technologies employed. Results of the commissioning of the feedboxes of the first LHC sectors are presented.
\end{abstract}

1 CERN, European Organization for Nuclear Research, Geneva, Switzerland

2 Institute for High Energy Physics, Protvino, Moscow region, Russia

3 Wroclaw University of Technology, Wroclaw, Poland

4 Fermi National Accelerator Laboratory, Batavia, USA

Presented at the 2007 Cryogenic Engineering Conference and International Cryogenic Materials Conference CEC-ICMC2007 16-20 July 2007, Chattanooga, USA

CERN

CH - 1211 Geneva 23

Switzerland 


\title{
DESIGN, PRODUCTION AND FIRST \\ COMMISSIONING RESULTS OF THE \\ ELECTRICAL FEEDBOXES OF THE LHC
}

\author{
A. Perin ${ }^{1}$, S. Atieh ${ }^{1}$, V. Benda ${ }^{1}$, A. Bertarelli ${ }^{1}$, A. Bouillot ${ }^{1}$, \\ K. Brodzinski ${ }^{3}$, R. Folch ${ }^{1}$, J. Fydrych ${ }^{3}$, M. Genet ${ }^{1}$, S. Koczorowski ${ }^{1}$, \\ L. Metral ${ }^{1}$, J. Miles ${ }^{1}$, F. Millet ${ }^{1}$, K. Polkovnikov ${ }^{2}$, R. Rabehl ${ }^{4}$, L. Serio ${ }^{1}$, \\ V. Sytchev ${ }^{2}$, V. Sytnik ${ }^{2}$, P. Trilhe ${ }^{1}$, R. van Weelderen ${ }^{1}$, V. Zhabitskiy ${ }^{2}$ \\ ${ }^{1}$ CERN, European Organization for Nuclear Research, 1211 Geneva 23, \\ Switzerland \\ ${ }^{2}$ Institute for High Energy Physics, Protvino, Moscow region, 142281, \\ Russia \\ ${ }^{3}$ Wroclaw University of Technology, Wyb. Wyspianskiego 27, 50-370 \\ Wroclaw, Poland \\ ${ }^{4}$ Fermi National Accelerator Laboratory \\ Batavia, Illinois, 60510, USA
}

\begin{abstract}
A total of 44 CERN designed cryogenic electrical feedboxes are needed to power the LHC superconducting magnets. The feedboxes include more than 1000 superconducting circuits fed by high temperature superconductor and conventional current leads ranging from 120 A to $13 \mathrm{kA}$. In addition to providing the electrical current to the superconducting circuits, they also ensure specific mechanical and cryogenic functions for the LHC. The paper focuses on the main design aspects and related production operations and gives an overview of specific technologies employed. Results of the commissioning of the feedboxes of the first LHC sectors are presented.
\end{abstract}

KEYWORDS: electrical feedbox, HTS current leads, superconductor, busbar, LHC

\section{INTRODUCTION}

The Large Hadron Collider, currently under commissioning at CERN, uses superconducting magnets to guide and focus the particles, protons or heavy ions, along the 
TABLE 1. List of the CERN supplied DFBs and their respective number of current leads

\begin{tabular}{lccccc}
\hline DFB type & $\begin{array}{c}\text { Number in } \\
\text { the LHC }\end{array}$ & $13 \mathrm{kA}$ & $6 \mathrm{kA}$ & $600 \mathrm{~A}$ & $120 \mathrm{~A}$ \\
\hline DFBA & 16 & 2 to 6 & 12 to 15 & 44 to 62 & 0 to 4 \\
DFBM & 23 & 0 & 3 to 5 & 0 to 1 & 4 to 12 \\
DFBL & 5 & 0 & 0 to 11 & 0 to 44 & 0 to 12 \\
\hline
\end{tabular}

$27 \mathrm{~km}$ of its ring. These magnets, located in a tunnel of a diameter of $3.8 \mathrm{~m}$ deep underground, operate either at $1.9 \mathrm{~K}$ in pressurized superfluid helium or in saturated helium at $4.5 \mathrm{~K}$. The electrical current of the magnets circuits connected to the electrical cryogenic feedboxes (DFB) range from 120 A to $13 \mathrm{kA}$. The feedboxes are located at both ends of the 8 sectors of the LHC for the arc magnets (16 DFBA), next to the magnets for standalone magnets (23 DFBM) and at the end of the superconducting links [1] where space in the tunnel does not allow a local installation of the DFBs (5 DFBL). The 8 feedboxes that power the final focusing magnets (DFBX) are part of the US contribution to the LHC and are described in [2]. In addition to being the main cryogenic-to-room temperature electrical interface of the LHC magnet system, the DFBs also ensure other essential functions like terminating the arcs and supplying some of the cryogenics for the magnets and the superconducting links. Providing these functions while being compatible with the diverse geometrical and functional constraints of the LHC required the development of specific configurations and technologies that are described in this paper.

\section{CURRENT LEADS AND BUSBARS}

The DFBs will power most superconducting magnets of the LHC. They integrate 1210 current leads for a total current carrying capability of about 2.8 MA. The number of current leads per DFB varies from 2 to 72 depending on the feedbox as is shown in TABLE 1.

The current is brought to the DFBs by copper cables and is transferred to superconducting busbars at $4.5 \mathrm{~K}$ by 4 types of current leads, designated by their current ratings: $13 \mathrm{kA}, 6 \mathrm{kA}, 600 \mathrm{~A}$, and 120A [3-6]. The $6 \mathrm{kA}$ and $13 \mathrm{kA}$ leads are installed individually on the DFBs, the $600 \mathrm{~A}$ are mounted in assemblies of four on a common flange and the $120 \mathrm{~A}$ are installed in assemblies of 4 or 8 on a common flange. The $13 \mathrm{kA}$, $6 \mathrm{kA}$ and 600 A use a High Temperature Superconductor (HTS) bottom part to limit the heat loads to the cryogenic system [3-5]. The bottom end of the HTS is connected to the $\mathrm{Nb}$-Ti based busbars at $4.5 \mathrm{~K}$ while the top part is kept at $50 \mathrm{~K}$ by a flow of $20 \mathrm{~K}$ helium that also cools the top part of the leads made of a copper heat exchanger. More than 1000 HTS leads are installed in the DFBs (see TABLE 2), making the LHC the world's largest

TABLE 2. Total number of current leads installed on the DFBs

\begin{tabular}{ccc}
\hline Type of lead & Total Number & Type \\
\hline $13 \mathrm{kA}$ & 64 & HTS / gas cooled \\
$6 \mathrm{k} \mathrm{A}$ & 258 & HTS / gas cooled \\
$600 \mathrm{~A}$ & 692 & HTS / gas cooled \\
$120 \mathrm{~A}$ & 196 & conduction cooled \\
\hline
\end{tabular}




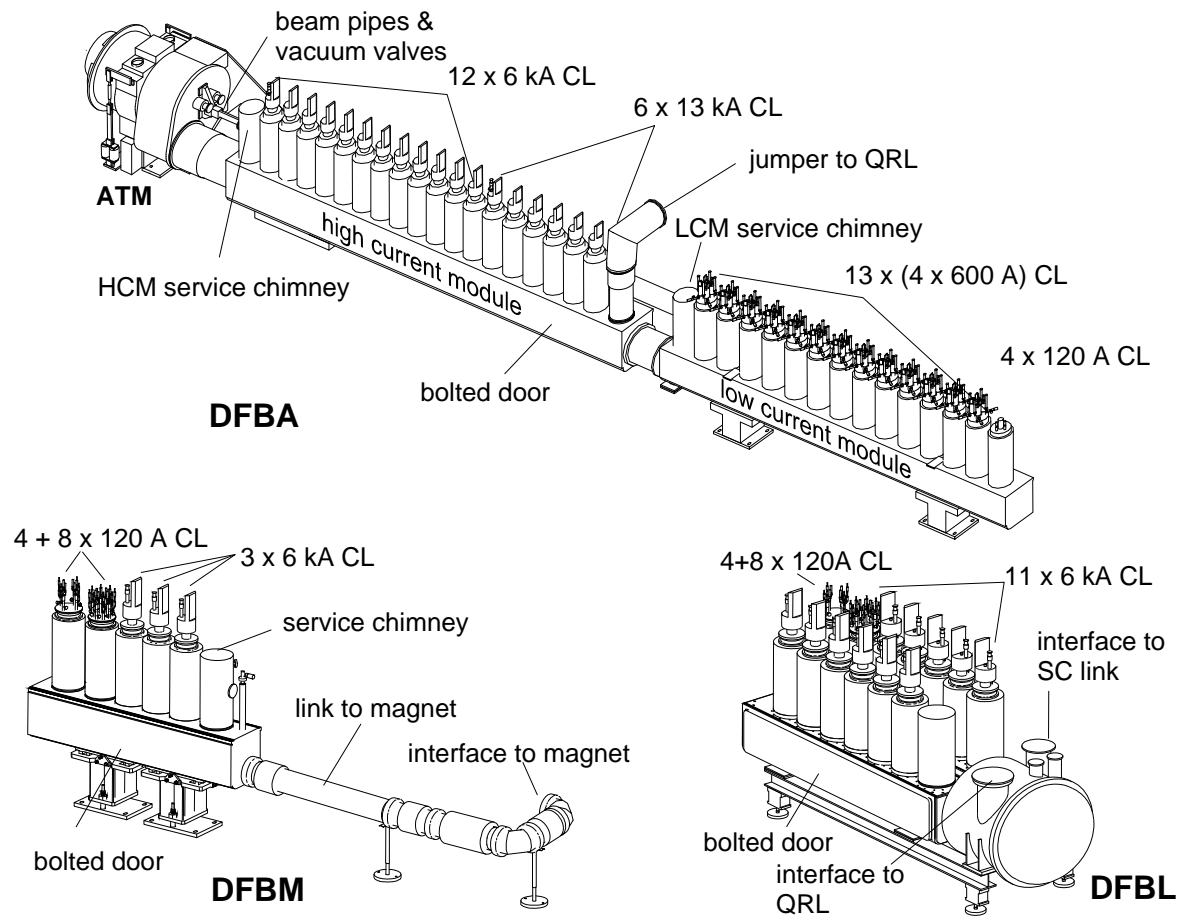

FIGURE 1. Typical configurations of DFBAs, DFBMs and DFBLs

user of HTS leads. More details concerning the development, performance and validation of the HTS leads can be found in [3-5]. The 120 A current leads are conduction cooled, with 3 gas cooled stages of heat intercept [6]. All voltage taps used to protect the superconducting circuits are installed directly on the current leads; this saves the instrumentation ports on the DFB and also allows changing a voltage tap with its lead in case of failure.

In the DFBs, the current is carried at $4.5 \mathrm{~K}$ and at $1.9 \mathrm{~K}$ by several types of superconducting busbars with cross sections varying from $2 \mathrm{~mm}^{2}$ for the $600 \mathrm{~A} / 120 \mathrm{~A}$ busbars to $320 \mathrm{~mm}^{2}$ for the $13 \mathrm{kA}$ busbars powering the main dipole magnets. All busbars are connected by low temperature brazing on one side to the current leads cables and on the other side to the magnets busbars. Because the design pressure of the magnets (20 bar) and of the DFBs (3.5 bar) are different, specially developed hydraulic separation plugs are installed on the busbars [7]. In the case of the DFBAs the plug also acts as a lambda plate between the $1.9 \mathrm{~K}$ superfluid helium volume and the $4.5 \mathrm{~K}$ helium bath of the DFBs. To obtain the required performance and robustness in case of thermal excursions, the busbars are bonded to a polymer plug with an epoxy resin. The seal between the plug and the busbar duct is realized by pressing the specially shaped plug onto a finely machined stainless steel flange [7].

\section{MECHANICAL AND CRYOGENIC DESIGN}

In addition to the electrical functional requirements, the mechanical and cryogenic design of the DFBs was driven by several factors that include among others the geometrical characteristics of the LHC tunnel, interface constraints, transport and installation requirements, design time, series production potential and ease of maintenance. The LHC tunnel with a diameter of 3.8 m leaves very little space for the installation of the 
DFBs or for the exchange of the leads. The slope of the LHC tunnel which varies from $0.36 \%$ to $1.39 \%$ and the requirement to realign the magnets connected to the DFBs while in cryogenic conditions were other important factors that also influenced the design.

A modular design was chosen: the DFBs are all made of current lead modules connected to the magnets by interface modules that cope with most of the requirements specific to each family of DFBs. FIGURE 1 shows the typical configurations of the three families of DFBs. The total length of the DFBs modules varies from $1 \mathrm{~m}$ for the shortest DFBM to about $11 \mathrm{~m}$ for the longest DFBA, the total mass being a few hundred kilograms and about 12 tons respectively.

\section{The Current Lead Modules and the Current Lead Cartridges}

All current leads are installed on two main types of modules: the High-Current Modules (HCM) which includes $13 \mathrm{kA}$ current leads, found on DFBAs, and the LowCurrent Modules (LCM) that do not include $13 \mathrm{kA}$ current leads and are part of most DFBs. The current lead modules are installed horizontally and the tunnel slope is compensated by the supporting system. The current modules are equipped with large bolted panels on the vacuum envelope that allow an easy access for exchanging the current leads. There are in total 16 HCMs and 45 LCMs in the LHC.

The current leads are installed in thin walled stainless steel chimneys as can be seen in FIGURE 2 (left). The bottom part of the lead is immersed in $4.5 \mathrm{~K}$ saturated helium. The $20 \mathrm{~K}$ helium is supplied to the lead through the chimney wall via an insulating ceramic pipe. To cope with the thermal contraction of the busbar cryostat that can reach $40 \mathrm{~mm}$ for the longest DFBs, the current lead chimneys are connected to the busbar duct by metal braided flexible hoses and the busbars are routed inside of the flexible hoses. As it is shown in FIGURE 2, the flexible hoses are configured in such a way that all hydraulic and electrical connections are brought close to the access door. The current lead, its chimney, inlet, outlet and busbar hoses is treated as a cartridge which can be exchanged as one unit without extracting the current lead. This operation only requires lifting the current lead cartridge by about $0.5 \mathrm{~m}$ and allows the exchange of leads even in the narrowest places in the LHC tunnel.

The principle of operation of the modules is shown in FIGURE 2: the level of the helium is regulated in a dedicated service chimney, the liquid helium is supplied to the chimneys by gravity through the busbar flexible hose and the evaporating helium cools the bottom part of the chimneys and exits from a specific outlet. Measurements performed on a simulated $13 \mathrm{kA}$ cartridge showed that the flow of evaporating helium provides an efficient cooling and can limit the conduction heat flow of the chimney to a few tens of milliwatts. As no connection is performed inside of the busbar cryostat, it is of reduced size and consists essentially of a manifold that distributes the busbars to the current leads, with a lambda plate or plug located at one of its ends.

\section{The DFBAs}

Most LHC superconducting magnets are installed in 8 continuous arcs of a length of about $3.3 \mathrm{~km}$. In order to power these magnets and extract the energy in case of a quench, one DFBA is installed at each end of the arcs, therefore also terminating mechanically and cryogenically the line of magnets. The 16 DFBAs integrate more than $70 \%$ of the CERN supplied current leads and have a combined current carrying capability of 2.1 MA. FIGURE 1 shows a typical DFBA. It consists of an Arc Termination Module (ATM) and two current lead modules. The second module is present only in DFBAs where the number 
of circuits is too large to fit into one module.

The current leads modules are placed beside the beam pipes that exit the arc at the ATM through special cold-warm transitions. The high current module is supported by the ATM and is linked on one side to the ATM through a connection that compensates for the tunnel slope and on the other side to the low current module. The HCM also ensures the interface of the DFBA with the cryogenic distribution line (QRL) [8] through a cryogenic jumper connection.

The ATM is the interface with the LHC arc, it withstands the forces resulting from vacuum and pressure, ensures the transition of the beam pipes to room temperature and includes a shuffling box that allows the re-routing of the busbars towards the current leads modules. The supporting system of the ATM allows the realignment of the magnets while in cold conditions. The shuffling box operates at $1.9 \mathrm{~K}$, and must withstand forces up to $60 \mathrm{kN}$ while ensuring the precise positioning of the beam pipes. Its supporting system includes a re-entrant stainless steel foot equipped with two level of heat sink at $60 \mathrm{~K}$ and at 4.5 $\mathrm{K}$ and a force relieving active support.

Due to the specificities of the different sectors of the LHC, all DFBAs although very similar have slightly different configurations.

\section{The DFBMs}

The standalone superconducting magnets in the matching sections of the LHC are powered by DFBMs. The 23 DFBMs of 13 different types integrate $16 \%$ of the current leads and have a combined current carrying capability of about $430 \mathrm{kA}$. A typical DFBM is shown in FIGURE 1. The DFBMs are located beside the magnet cryostats and are connected to the magnet through an articulated link that allows the realignment of the magnets in cryogenic conditions. They consist essentially of a current lead module with its own supporting system. They do not require a dedicated jumper to the QRL, the cryogenic fluids are supplied trough the magnet cryostat.
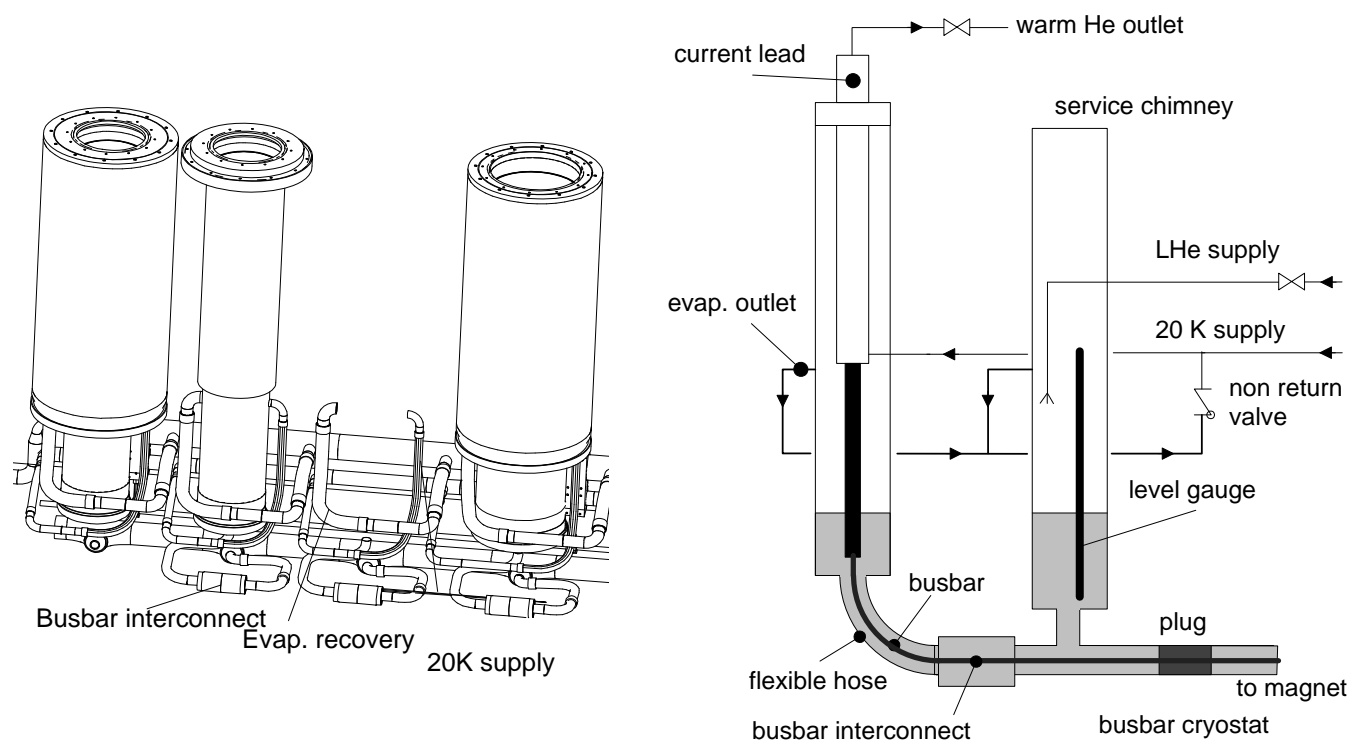

FIGURE 2. Scheme showing the configuration of the cartridges in the DFBs (left) and the cryogenic principle of operation of the current lead modules (right). 

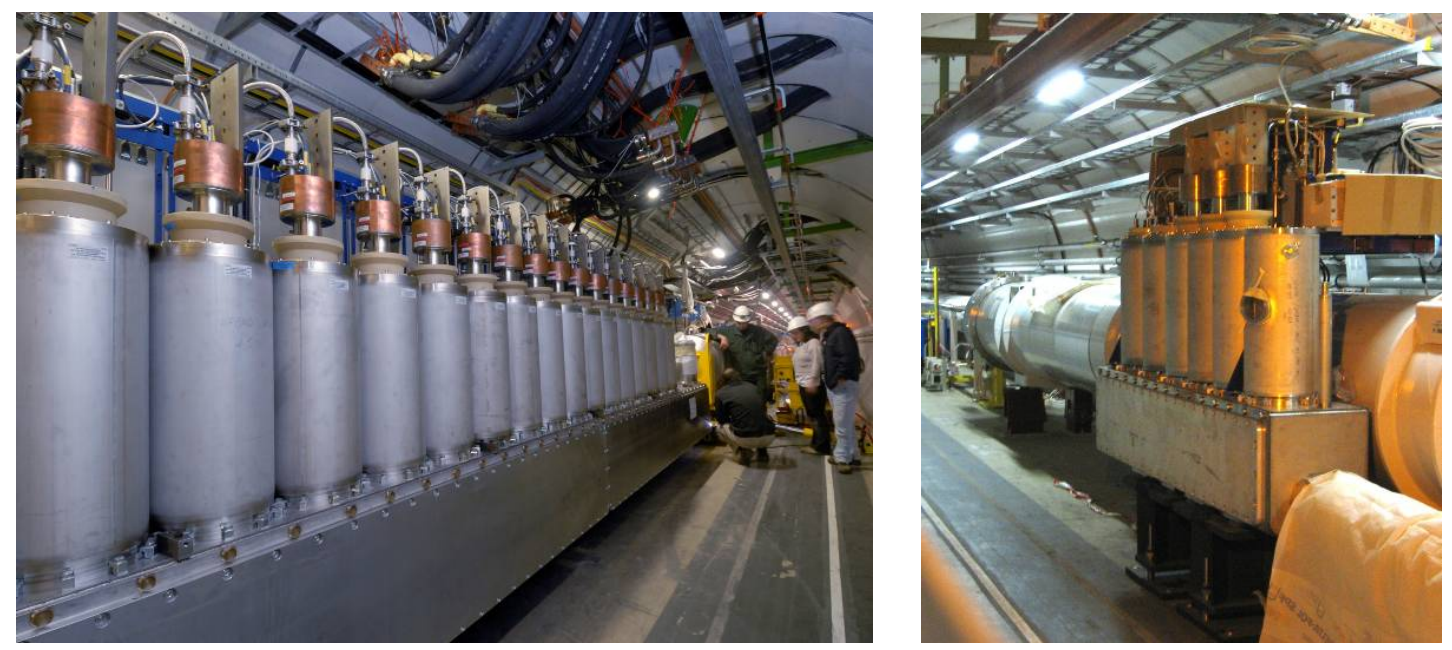

FIGURE 3. pictures showing the DFBA powering one of the sides of sector 7-8 (left) and a DFBM powering one of the standalone magnets (right) installed in the LHC tunnel

\section{The DFBLs}

When the configuration of the LHC tunnel does not allow the installation of the power supplies close to the superconducting magnets, powering is performed with superconducting links [1] connected to DFBLs. The 5 DFBLs of 2 different types integrate $10 \%$ of the current leads and have a combined current carrying capability of about $310 \mathrm{kA}$. FIGURE 1 shows a typical DFBL. It consists of two LCMs installed side by side and connected together with an interface module. The interface module also ensures the connection to the QRL and to the superconducting link. Four out of five DFBLs also include a sub-cooler to supply $4.5 \mathrm{~K}, 3$ bar sub-cooled helium to the superconducting links while one DFBL uses the superconducting link in a reverse fashion for the supply of its cryogenic fluids.

\section{PRODUCTION OF THE FEEDBOXES}

After two pre-series DFBA were produced by CERN, the final assembly of the series DFBs was performed in about 12 months from May 2006 to May 2007, with a production rate of about 5 modules/month which corresponds to about 6 leads installed and connected per working day. This production was the result of an intense collaboration between several groups of CERN, the IHEP Institute of Protvino, Russia and several CERN contractors. Thanks to the modularity of the design, the assembly operations were split in several production lines for: the current lead cartridges, the busbar system, the arc termination modules, the HCMs and the LCMs. A dedicated logistics team was set up to handle the more than 10000 components. FIGURE 3 shows a DFBA and a DFBM installed in the LHC tunnel.

A major issue for the production was the capacity to assemble and weld thousands of components with very stringent leak tightness and mechanical requirements. The effort started with the careful selection of the materials and welding configurations. Even the basic elements were all validated individually, in particular for welded components. Rejection rates of more than 50\% were sometimes found for commercial components like pipe elbows. During the assembly phase, the welding operations were checked with the 
utmost attention, with specific validations for personnel and welding methods. The components and sub-assemblies were tested $100 \%$ for leak tightness after each production step, and a minimum rate of $10 \%$ X-ray inspection was kept for all welds until the end. The result of this major effort was that no internal leak above the specified value was found during the final leak and pressure tests.

Another essential aspect was the quality of the brazed connections of the busbars. As they could not be tested at room temperature, each operator was regularly re-qualified during the production and samples were systematically measured at low temperature to ensure a constant quality. The integrity of the insulation was also checked at each assembly step.

All DFBs were pressure tested and electrically qualified with their hydraulic and electrical ancillary equipment prior to their installation into the LHC tunnel.

\section{FIRST RESULTS FROM COMMISSIONING}

Starting from April 2007, the DFBs of sector 7-8 of the LHC have been cooled and their circuits progressively powered [9-10]. One of the first tasks during the commissioning was to verify the liquid helium levels in the DFBs and the correct cryogenic operation. For this purpose several DFBs of sector 7-8 were equipped with level sensors not only in the service chimneys, but also in some current leads chimneys. The correct operation of the cryogenic configuration, in particular concerning the relative levels in the current leads chimneys, was checked during a boil-off test on a DFBA HCM. The liquid level probes in the service chimney and the $13 \mathrm{kA}$ chimney are located $4.9 \mathrm{~m}$ apart. A stable liquid level from one end of the current lead module to the other is measured to within $5 \mathrm{~mm}$ of agreement, which includes a $\pm 5 \mathrm{~mm}$ accuracy of the probes, and a few $\mathrm{mm}$ of accuracy of construction and in the final horizontal placement of the DFB in the tunnel. A boil-off test also provided an opportunity to calculate the total heat load to the $4.5 \mathrm{~K}$ bath. For the DFBA HCM of IR8 and its eighteen current lead chimneys (12 x $6 \mathrm{kA}$ and $6 \times 13 \mathrm{kA})$, the heat load at zero current is about $13 \mathrm{~W}$, well inline with the estimated values.

All of the DFBs in the LHC sector 7-8 have been released for powering. Milestones to date include the DFBMs powering the magnets in the matching sections up to currents of 6000A and the DFBA HCM powering the sector 7-8 arc quadrupoles up to $6500 \mathrm{~A}$ (nominal current is 11870 A). During all commissioning operations the HTS current leads

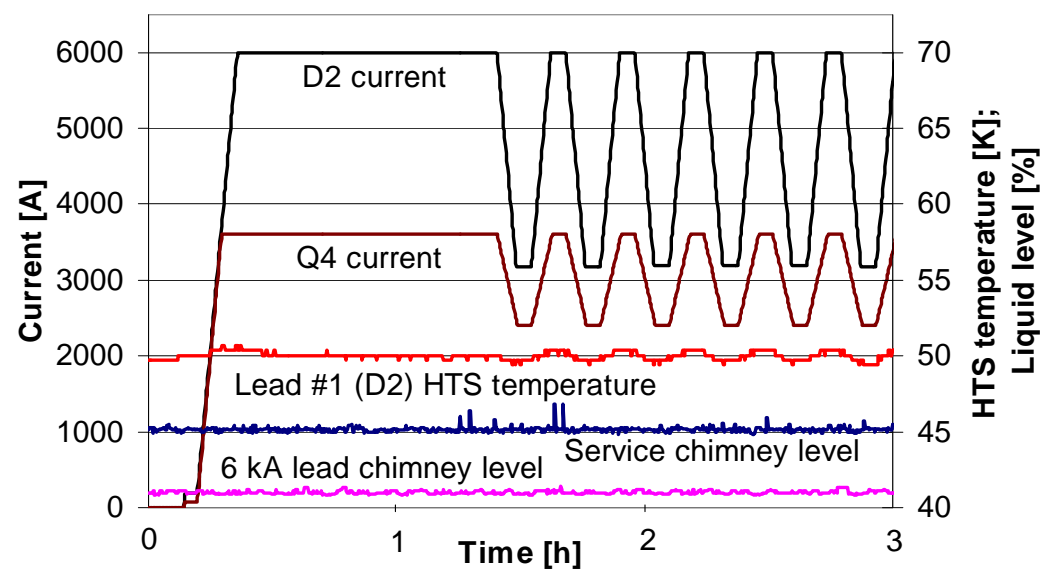

FIGURE 4. liquid helium levels in a DFBM powering a dipole (D2) and a quadrupole (Q4) magnets (1\% corresponds to $4 \mathrm{~mm}$ for the service chimney and to $1.5 \mathrm{~mm}$ for the $6 \mathrm{kA}$ chimney) and temperature of the top part of the HTS during current cycling. 
exhibited a nominal performance, with parameters very close to the measurements performed during validation tests. The liquid helium levels and temperatures could constantly be controlled well within the powering limits for all DFBs during the current cycles required by the magnetic system commissioning operations, as can be seen in FIGURE 4 for the DFBM powering the DFBs.

\section{CONCLUSIONS}

Powering the superconducting magnets of the LHC arcs and matching sections is performed by 44 cryogenic electrical feedboxes of three main families that integrate 1210 current leads. The DFBs also ensure cryogenic and mechanical functions for the LHC arc magnets and for the superconducting links of LHC. The 44 DFBs were developed and successfully produced and are now being commissioned. The first results from commissioning show cryogenic parameters well within the operating limits and a nominal performance of the HTS current leads.

\section{ACKNOWLEDGEMENTS}

The authors gratefully thank all the personnel of CERN, of IHEP and of CERN external contractors for their constant and efficient collaboration during the design and production of the DFBs.

\section{REFERENCES}

1. Goiffon T., Lyngaa J., Metral L., Perin A., Trilhe P., van Weelderen R., "Conceptual design of the Cryogenic Electrical Feedboxes and the Superconducting Links of LHC" in Proceedings of ICEC 20 conference, Beijing, China 2004, edited by Zhang L., Lin L, Chen G, pp. 1059-1062 (2005)

2. Zbasnik J., Corradi C, Gourlay S., Green M, Hafalia A., Kajiyama Y, Knolls M., LaMantia R., Rasson J., Reavill D., Turner W., IEEE Trans. Appl. Supercond. 13, pp. 1906 (2003)

3. Ballarino, A., IEEE Trans. Appl. Supercond. 12, pp. 1275-1280 (2002)

4. S.A. March, C. Beduz, A. Ballarino and Y. Yang, "Full Cryogenic Test of 600 A HTS Hybrid Current Leads for the LHC", M.K. Al-Mosawi, , Proceedings of ASC 2006, August 2006, Seattle

5. S.Turtu, A. della Corte, A. Di Zenobio1, R.Viola1, M.Napolitano, C. Fiamozzi Zignani, J. Mayorga, U.Besi Vetrella, A. Ballarino, P. Chambouvet, "Cryogenic Test of High Temperature Superconducting Current Leads at Enea" in Advances in Cryogenic Engineering, 51, edited by by J. G. Weisend II

6. A. Ballarino, LHC project document LHC-DFL-ES-0002, CERN

7. Marie R., Metral L., Perin A., Rieubland J.-M., "Measurement of the thermal properties of prototype lambda plates for the LHC", in Proceedings of ICEC 20 conference, Beijing, China 2004, edited by Zhang L., Lin L, Chen G, pp. 1039-1042 (2005)

8. Riddone G., Trant R., "The Compound Cryogenic Distribution Line for the LHC : Status and Prospects" in Proceedings of the 19th International Cryogenic Engineering conference edited by Gistau-Baguer, G, Seyfert, P. , pp. 59-62 (2003)

9. F. Millet et al., "Commissioning the cryogenic system of the first LHC sectors", paper presented at this conference

10. L. Serio et al., "Validation and performance of the LHC cryogenic system through commissioning of the first sectors", paper presented at this conference 